\title{
A Study on Pig Slaughter Traceability Solution Based on RFID
}

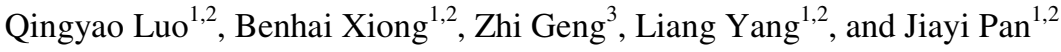 \\ ${ }^{1}$ Institute of Animal Sciences, Chinese Academy of Agricultural Sciences, Beijing 100193, \\ China \\ ${ }^{2}$ State Key Laboratory of Animal Nutrition, Beijing 100193, China \\ ${ }^{3}$ Animal Husbandry and Veterinary Bureau, Tianjin 300210, China
}

\begin{abstract}
Due to an assembly line production and poor environment conditions in pig slaughterhouses, collection of slaughter tracing information is not a simple thing. Based on the UHF radio frequency identification (RFID) technologies, this study designed a RFID tag for carcass, a RS232-PS2 data conversion line and some data norms such as the RFID carcass tag and partition meat label norm, and developed online reading and writing system for RFID tags, accomplished RFID identification for carcass and automatic identification on the slaughter line. Through identifying ear tags at the pig heading process, fixing RFID carcass tags at the half-carcass process, printing partition labels at pork exclusive stores, the study were able not only to collect, transmit and deal traceability information for pig slaughter in the key processes of the whole pig slaughter line, but also print a set of commercial cutting meat tags of 1D bar code based RFID carcass tag in the sales store. This study has been applied for demonstration in Tianjin and explored any possibility for application of RFID technology in pork quality traceability system from both technology and application links.
\end{abstract}

Keywords: Pig, RFID, Traceability, Automatic Collection.

\section{Introduction}

Since a case of the Bovine spongiform encephalopathy (BSE) occurred in Britain in 1986, zoonosis (BSE), highly pathogenic avian Influenza (HPAI) and food quality safety problems such as Sudan red powder, inferior milk powder happened occasionally and so have been paid wide attention[1-2].The first quality traceability system for animal products in China was the animal immune identification system began in 2002[3]. Following Animal Industry Act in 2005, the ministry of agriculture has promulgated "The measures on the administration of animal identification and farming files" ("the administrative measures") [4], which put in effect on July 1, 2006. Implementing the administrative measures begins mainly to construct the animal quarantine traceability system and gradually to establish the traceability system of animal products quality and safety. The animal quarantine traceability system, the demonstration project of the ministry of agriculture, mainly adopted 2-D barcode technologies to identify animal individuals, took IC cards as mobile data carriers for animal quarantine, conducted identification in the cold-chain transportation, 
subdividing and packaging links and collection, transmission and switching of the traceability data by the GPRS communication, PDA identification devices and the remote central database[5-8]. This program mainly solved how to establish electronic farming files in the pig farming link, how to take the supervision and collect data in transportation of live pigs. But systematic official regulations for information collection in the swine slaughter link and the pork sale link was not found due to different administrative departments. Therefore, technically to explore reliable identification technologies of intermediate-to-end pork products such as the slaughtering link and data collection technologies such as core technologies about internet of things is an important content of constructing comprehensive tracking and traceability system for animal products, especially pork products in China.

Radio frequency Identification (RFID) is a contactless automatic identification technique and can identify target object automatically via radio frequency signal and obtain relative data. Based on the working frequency, it is divided into: low frequency system (LH,124 134KHz) with the characteristics of stable technical performance, strong penetrability and diffraction, cheap cost, but short reading distance; High frequency system $(6 \sim 13.56 \mathrm{MHz})$ with a weak penetrability and diffraction, a long recognition distance, a better anti-collision ability, identification speed quicker than LH, bigger environmental impact; Ultrahigh frequency (UHF, 840 960MHz) RFID system with a good anti-conflict ability, strong penetrability, many times reading and writing, big memory capacity, low cost for passive tags, small volume, high reliability and long life, tolerance of poor environments, and convenient for using in assembly line[9-10].In practical applications, the earliest reports were respectively LF RFID chip implanted in piglets until the sale[11], or identifying pork carcass[12], UHF RFID $(13.56 \mathrm{MH} \mathrm{z})$ applied in the slaughter link in a beef traceability system[13]. But, as to most of present assembly lines in pig slaughterhouses, environments were often worse than beef processing slaughtering line, such as dark and damp, and workers with a low cultural level were difficult to ensure entirely corrected identification if bar code identification was used and more manual intervention required. As for a LF or HF system, an effectively automatic identification and data collection system was not found up to now because of special characteristic of the slaughter line. Thus, this paper developed an UHF RFID data collection system being able to identify pig carcass and to get identification data automatically and continuously, and a pork quality traceability platform with the collection of identification data in the pig slaughter link, and realized an association with the breeding files record system in the pig breeding link and the data query system in the sale link, in order to build a tracking and tracing platform for the quality and safety of pig, and to explore a high efficient, reliable data collection solution in the pig slaughter link based on internet of things.

\section{Material and Methods}

\subsection{Study Object}

This study took pig slaughterhouses in Tianjin as a study model, pig and products as study objects and explored the collection solution of the slaughter traceability information of commercial pigs. 


\subsection{Traceability Ear Tag}

According to the administrative measures stipulated by the ministry of agriculture, all pigs are required to wear a traceability ear tag with a unique identification number in the first vaccination, and establish breeding files (electronic or paper files) in the farm. When commercial pigs were purchased by pig slaughterhouses, traceability ear tags, the original area quarantine card and the vehicle disinfection certificate card were all checked. Only qualified pigs were purchased.

Identification code number According to the administrative measures, the unique identification number for pig consists of 15 digits, in which the first digit represents the type of animal, namely 1 for pig, 2 for cattle, 3 for sheep. The following 6 digits represent the administrative division code which division pig breeding farmer is located in and in line with the GB T2260-1999 (Dec 31, 2006)[14]. 8 digits from eighth to 15th represent an individual sequence number of the same type of animal breeding in the same administrative division. For example, a code number as "1 12011100006008 " means that breeding animal type is pig and the breeding administrative division is Xiqing district (120111), the sequence number of the individual pig is 6008. If the last number is not 8 digits, it should be supplemented to 8 digits by zero in order to make the code number up to 15 digits.

Traceability Ear tag Based on the above code rules, the type of ear tag as figure 1 was designed. A kind of special 2D barcode is engraved on top of the ear tag, which is identified by the special code reader. On both sides of the $2 \mathrm{D}$ barcode figure, there are code digits being able to be identified by eyes. On the top of ear tag is a $30 \mathrm{~mm}^{2}$ of circle area, the distance from the circle area at the top to the small round cone at the bottom is $25 \mathrm{~mm}$. The area of $2 \mathrm{D}$ barcode figure is $12 \times 12 \mathrm{~mm}^{2}$.

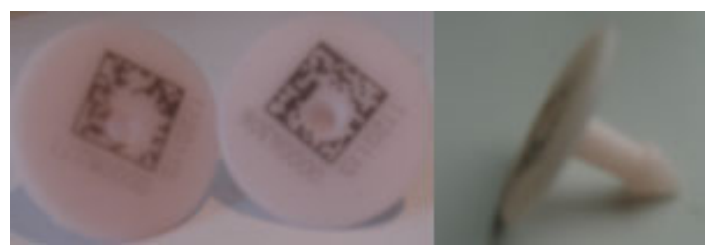

Fig. 1. The special 2-D bar code tag used in China

Traceability devices for the ear tag The ear tag is key identification information for breeding information, moving information and monitoring information during pig production, and accurately collecting the pig ear tag information is required in order to make an association between the pig slaughter traceability information and the former pig breeding information.

Traceability Device for ear tag: Although digits on the traceability ear tag can be seen by eyes, 2-D bar code can only be identified by the special reader device with the 2-D decoding procedure. Two intelligent mobile PDA devices are authorized by the 
animal traceability administrative, the ministry of agriculture by the public bidding, which can identify the traceability ear tag for pig with a camera (fig. 2) [5]. The left PDA is based on Microsoft Windows mobile 6.0 and the right on Linux system. Information of ear tags can not only directly upload, but also temporarily save in the IC inserted into PDA devices.

In the fig. 3, there is a barcode scanner identifying 2-D barcode on the tracing ear tag, fit for the workplace with a computer terminal. Ear tag information identified not only saved in the connecting computer terminal, and also uploaded to a server database by the intranet. Its identifying speed and effect are better than that of the former two mobile intelligent PDA.
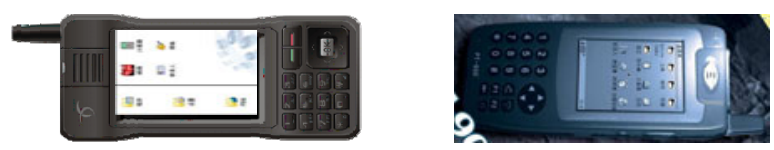

Fig. 2. Two types of PDA with reading camera

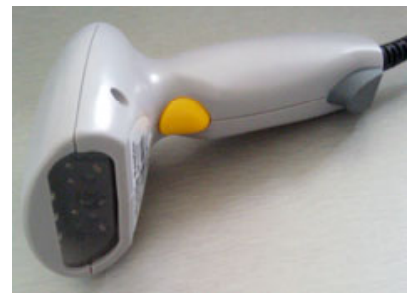

Fig. 3. Barcode scanner identifying the special 2-D barcode tags

\subsection{RFID Carcass Tag}

Pork carcass is intermediate products in the pig meat production chain, is a connecting link between pig individuals (pig breeding) and pork partition products (pork sales). Reasonably to identify carcass is a key part of realizing the whole pork production traceability.

RFID code number the cod number is 20 digits, the first 6 digits is the administrative code, 2 digits from the 7 th to the 8 th represent the sequence number of pig slaughterhouse in the administrative district, the following 8 digits show the date digit (yyyymmdd), the last 4 digits are the serial number of the same batch of slaughtering pig in the slaughter house. The most number of each batch slaughtering pigs is 9999. For example, as for 32002101201003270001 , "320021" is the administrative code, "20100327" means that the slaughtering date is march 27,2010 , "01" and "0001" respectively for sequence number of the slaughterhouse in the administrative district and pig individual in the slaughtering batch. 
RFID tag Because there is an assembly line production in the pig slaughterhouse and the processing work is also in worse environments, adopted tags are required to be waterproof and to be able to read and write quickly. RFID tags was finally designed after many times of write and read test and waterproof test. In order to ensure write and read quickly in the line production, size of RFID chip was required to be considered. At the top of the tag was a rectangle hole for fixing tags.

RFID reader and antenna UHF RL9001 RFID reader this RFID reader as shown in fig. 4 (left) is able to write and read electric tags according to ISO-18000-6B and EPC CLASS1 G2, which is widely used in data collection system for animal tracking and tracing, monitoring and controlling of logistics, manufacturing automation management. The reader adopts aluminum alloy shell and is able to install in indoor enclosure or outdoor protective box with good ventilated, dustproof and rainproof condition. Its working frequency is $902 \mathrm{MHz}-928 \mathrm{MHz}$, the effective read distance is more than 5 meters and the write distance more than 3 meters, reading and writing time each single-byte is respectively $6 \mathrm{~ms}$ and $50 \mathrm{~ms}$.

UHF RL9021 RFID flat reader As shown in fig. 4 (right) is developed for pork sales counters in pork product traceability system, data communication interface with built-in antennas is RS232, the distance for reading tag is less than $10 \mathrm{~cm}$, the writing distance is less than $5 \mathrm{~m}$. In order to cooperate with printing the pork partition label, RS232-PS2 data conversion line has been developed, by which data information collecting by RFID reader transmits to the partition printer named as "Dingjian" to print 1-D barcode partition label corresponding with RFID carcass tags.
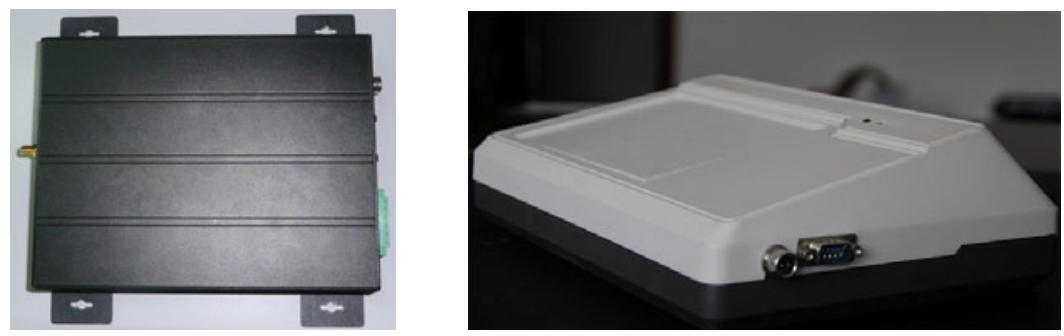

Fig. 4. UHF RFID reader

\subsection{Partition Tag}

Partition tags mainly include tag information at the top of the tag, products description information at the middle including product name, batch, date, company, carcass barcode and visible digits, traceability style prompt at the bottom. In the above tag, carcass code firstly identified by UHF RFID reader and then transmits to barcode printer through RS232-PS2 data conversion line, finally the printer print out 1-D barcode and digits according to carcass code number. "Product name" is usually selected and then print out partition labels through the manual buttons. 


\subsection{Software Architecture for Slaughter System}

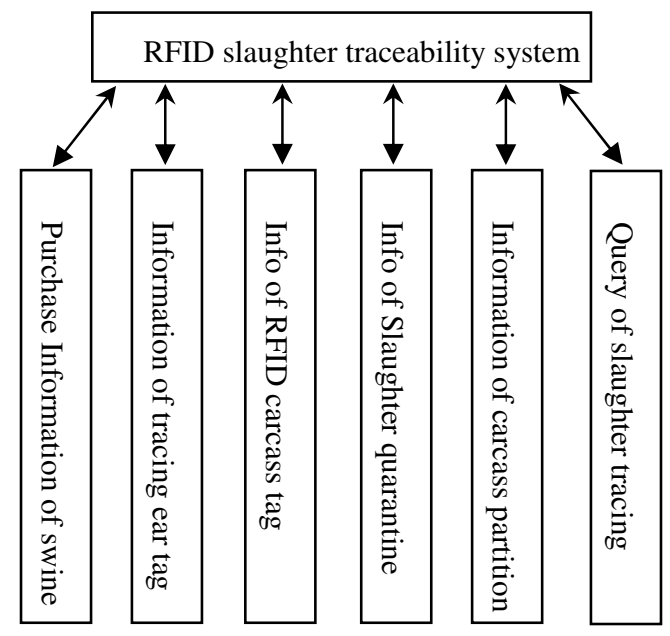

Fig. 5. Framework of slaughter system based on RFID

\subsection{System Development Tools}

System development is based on Microsoft Windows Enterprise Server 2003, main tool soft wares are respectively Microsoft Visual Studio 2005, Windows Mobile 6.0 SDK, Microsoft ActiveSync 4.5, Microsoft SQL Server 2005, Microsoft Visual Basic 6.0, etc.

\section{Results and Discussions}

\subsection{Collection of Traceability Ear Tag Information}

A computer furnished with "Slaughterhouse ear tag information collection system" (fig. 6) and a 2-D bar code scanner (fig. 3) were arranged at the heading process on the slaughterhouses line and connected to the slaughter traceability database by the intranet.

The worker at the heading process uses the scanner to identify barcode information with ear tags and upload to the slaughter traceability data center while heading and cutting off ear tags.

During scalding process, ear tags sometimes lose. In order not to impact normal running of the slaughter assembly line and to ensure integrity of tracing information, the ear tag information system produces an alternative ear tag code for the pig lost its ear tag and upload to the slaughter tracing data center [15]. Because any pig slaughterhouse slaughter in turn based on suppliers of commercial pig during purchasing and slaughtering commercial pigs, the accuracy of information for origins or owners of commercial pigs will not be affected. 


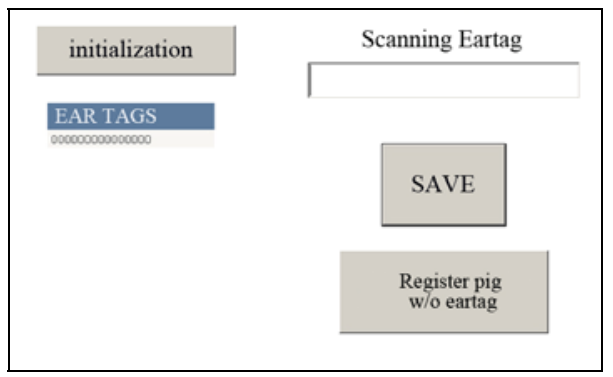

Fig. 6. Interface for Writing UHF RFID

\subsection{Fastening Carcass Tag}

Fixing RFID tag is located at the half-carcass process, where a computer with "RFID tag read and write system" (fig. 7), UHF RFID reader and antenna are assigned. The computer connects to the slaughter tracing center by the intranet.

Before slaughter, the antenna is first adjusted to suitable location, then the computer is turned on and "read and write system for RFID tag" is automatically started. The system obtains ear tag numbers in sequence from the slaughter tracing database and generates RFID tag number according to RFID carcass metadata norm. During slaughtering, the worker firstly put RFID tag in front of the RFID antenna and makes the tag code written, then fixes it in the front-end of the half carcass and finally put the carcass on half partition machine to make it into the half carcass. Lashing used is a hard plastic belt with tooth and ring by one head fastening with the other one through the tag hole and round the small hole in the front-end of the carcass.

According to requirements of the slaughterhouse, the relationship of ear tags with carcass tags is not only 1 to 1 , but also 1 to 2 . Due to ensure reading and writing speed for tags and decrease potential errors, only 20 digits of RFID carcass tag is written into RPC district of RFID tag in the system and the others all into the slaughter tracing database.

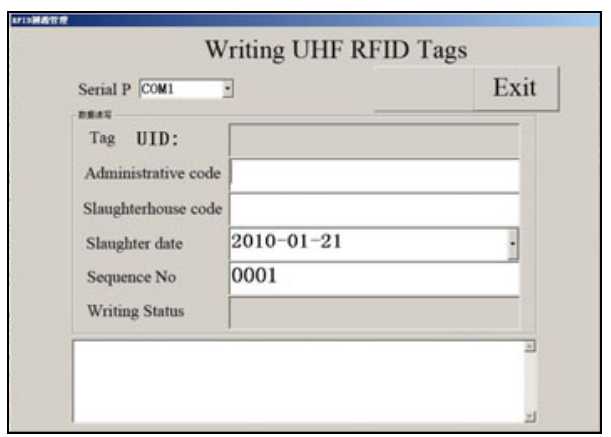

Fig. 7. Interface for Writing UHF RFID 
RFID tags are made by two ways: one is that RFID carcass tags are written by date and sequence before slaughter, and fastened on the carcass in turn during slaughter. The other is that RFID carcass tags are written and fastened during pig slaughter.

In general, after slaughter, the official veterinary or quality supervisor at the slaughterhouse firstly check in information for ear tags, carcass tags and carcass quarantine and then submit all information the pork traceability center. While submitting, a slaughter event for every carcass is produced according to the slaughter tracing metadata standard and then uploads to the pig traceability database center.

\subsection{Partition Label and Query}

According to systematic design, an ultrahigh frequency (UHF) flat RFID reader, a "Dingjian" barcode printer and a touching screen query machine are arranged in the pork exclusive store. During the process, the RFID carcass tag hung on the half carcass is red at first by the flat RFID reader. And the carcass tag information obtained (20 digits) is transmitted to the barcode printer for carved meats on time by the RS232-PS2 conversion data line, then select pork carving classification and type "printer" button, the printer can print 1-D barcode label based on the RFID tag, which includes pork position, 1-D image and visible digits for the carcass code and name of sale company for the products. Pork classification in this study consists of lean meat, soup bone, vertebra bone, marbled meats with skin, the front shoulder meat with skin, the ear leg meat with skin, whole lean meat, shoulder butt, pig elbow, west steak, pork chop, pork spare rib bone and so on.

When a partition label is put in front of the scanner on the touch screen query machine and scanned, the tracing query system searches on the pork quality tracing center. If the relative pig ear tag and carcass tag are existed, information for pig breeding farm, pig slaughterhouse and official supervisions is respectively shown on the screen. This study developed various query styles of query and short message service based on mobile devices such as PDA, mobile phone and so on, which is greatly convenient for pork consumers' right to know.

\section{Conclusion}

Quality traceability of pork products begins with pig breeding until dining-table of consumers and involves many segments. Influencing factors are complicated; a variety of data is required to be collected and perceived. At present, it is very difficult to collect and carry out whole traceability information. Therefore, only information for ear tags at removing ear tags and RFID carcass tags at halving pig carcass process is collected based on analysis of traceability key points, especially on intermediate slaughter processes. That is, the study not only collected wholly and normatively processed slaughter traceability information, and also made one-one relation between pig breeding process information and terminal sales information according to the systematic design, realized the traceable query of pork quality and safety. This study is a subsystem of the traceability system for quality and safety of swine products, mainly to reduce or avoid phenomenon that easily occur or make mistakes between material chain and information chain in tracing system for animal products' quality by things 
internet technologies, i.e. UHF RFID, that not only ensures the integration of tracing information and improves the effectiveness of tracing information query [5-7]. Now, this data collection system for slaughter link has applied to the traceability platform for quality and safety of swine products in Dongying city in Shangdong province, Tianjin city and Hebei province [15-18].

Collecting solution of key data in slaughter link is all responsible by the official veterinarian. Data may be collected and uploaded by the slaughterhouse technologist who must be authorized by the official vet responsible for the authenticity. Obviously, this mode combines present official veterinarian and meat quality traceability system, which is currently a temporary solution without an independent third-party certification. Expected effects have obtained through some cities' demonstration. However, the responsibility, right and interest need to be solved by institutional construction.

Application of RFID technology in slaughterhouses traceability system, helpful to ensure automation, reliability and validity of collecting pig slaughtering traceability information during the pig slaughter process, solved technical challenges of information collection in a harsh environment of pig slaughtering and reached technical effects better than the low frequency and high frequency RFID technology. This shows that UHF RFID technology has wide application prospects in the slaughtering link for pig slaughtering and other animal slaughtering. But there are still some problems to pay attention[9].

The first is how to unity articles coding and frequency. If code or frequency applying in food supply chains is disunity, it must result in a lot of inconvenience for arrangement of reading and writing devices, query, tracking and tracing. Because so called ultra high frequency is actually involved with a wider scope of frequency interval, i.e. $860 \sim 960 \mathrm{MHz}$ [19-20], which specific frequency is used need discuss extensively and avoids conflicting with other industries. The second is the cost and recycle of RFID tags. If every single product, including immediate products such as pork, beef , is able to use RFID tags, this will inevitably require that price of tags is reasonable, tags is able to recycle, the identifying cost every carcass reduced to a certain acceptable degree for all pig slaughterhouses. Passive chips used in this system adopted the imported inlay chip and its price is relative high, so it is just for a small demonstration application. Once substantive demonstration put in effect, price of tags require reduce to an acceptable balance.

Although large-scale application of RFID tags in food safety field has a long way to go, with the rapid development of digit and information technology, the tendency that identification means based on RFID and the global unity of coding system together with HACCP system are applied in food safety and traceability system, is invariable. For example, EU has a regulation early, all beef products in EU markets must have electronic identification since 2005 [1-2]. Therefore, to save something for a rainy day, to start with new technology research as early as possible, to provide better service for control and supervision of food safety, to supply more safety and relieved food for consumers is the only way.

\section{Acknowledgements}

Appreciation is extended to $\mathrm{Wu}$ Weiping, for his practical suggestions and helps in process of this system development. Funding were provided by the national high 
technology research and development program of China (863 Program) (2006AA10Z266), Tianjin municipal science \& technology Innovation fund (06FZZDNC0100) and the national key technology R\&D program (2006BAK02A16).

\section{References}

1. Fu, L.T., Xiong, B.H.: Animal Identification and Product Traceability Technology, pp. 1-2. Chinese Agricultural Science \& Technology Press, Beijing (2009) (in Chinese)

2. Lu, C.H., Wang, C.J., Hu, Y.N.: Animals and their Products Identification Technologies as well as Traceability Management, pp. 46-47. Chinese Agricultural Science \& Technology Press, Beijing (2007) (in Chinese)

3. The Measures on the Administration of Animal Quarantine (in Chinese), http: / / www.gov.cn/gongbao/content/2003/content62566.htm

4. The Management Method of Livestock and Poultry Identification and the Farming Files (in Chinese), http://www.agri.gov.cn/blgg/t20060628_638621.htm

5. Xiong, B.H., Lin, Z.H.: A Solution on Pork Quality Safety Production Traceability from Farm to Dining Table-Taking Tianjin City as an Example. Agricultural Sciences in China 42(1), 230-237 (2009) (in Chinese)

6. Xiong, B.H., Fu, R.T., Lin, Z.H.: Identification of Swine Individuals and Construction of Traceability System. under Free-range Pig-rearing System 25(3), 98-102 (2009) (in Chinese)

7. Xiong, B.H., Fu, R.T., Lin, Z.H., Luo, Q.Y., Yang, L., Pan, J.R.: A Solution on Pork Quality Traceability from Farm to Dinner Table in Tianjin City, China. Transactions of the CSAE 9(1), 147-156 (2010)

8. Ma, C.G.: Overall monitoring of pork industrialization production and development of traceability system. Transactions of the CSAE 24(9), 121-125 (2008) (in Chinese)

9. Zhao, J.Y., Tao, L.L., Gao, S.Z.: Studies on Animal Food Safety Traceability System Using RFID Technology. Journal of Yunnan Agricultural University 23(4), 528-531 (2008) (in Chinese)

10. RFID World website: Strengthen RFID System of Animal Identification and Disease in Ningxia (in Chinese),

http://info.secu.hc360.com/2009/10/101112167329.shtml.

11. Company Chunyuan: Implanting RFID chip in ear of pig and Realizing breeding information (in Chinese),

http: / /www.rfidchina.org/rfid-info-5134-182.html.Feb13, 2007

12. Lu, C.H., Wang, l.F., Xie, J.F.: Design of Digital Traceability System for the Safety of Factory Pork Production. Jiangsu J. of Agricultural Sciences 20(4), 259-263 (2004)

13. Zan, L.S., Zheng, T.C., Shen, G.L.: Design and Development of Quality Traceability Information Management System and Safety of the Beef Production's Entire Processes. Agricultural Sciences in China 39(10), 2083-2088 (2006) (in Chinese)

14. National Bureau of Statistics of China (NBSC): Codes of administrative regions of the People's Republic of China (2006) (in Chinese)

15. Yang, L.: The design and realization of slaughter technology in the system of pork quality safe traceability. Agriculture Network Information (1), 22-25 (2008) (in Chinese)

16. Xiong, B.H., Lu, J.Q., Yang, L.: Development for Breeding Performance Management System on Pig Farms. In: Computer and Computing Technologies in Agriculture, vol. 1, pp. 267-275 (2008) 
17. Xiong, B.H., Fu, R.T., Lin, Z.H., Luo, Q.Y.: A Solution on Identification and Rearing Files in Smallhold Production. In: Computer and Computing Technologies in Agriculture, vol. 3, pp. 2249-2258 (2009)

18. Luo, Q.Y., Xiong, B.H., Yang, L.: A Collection Scheme for Tracing Information of Pig Safety Production. In: Computer and Computing Technologies in Agriculture, vol. 3, pp. 2239-2247 (2009)

19. Xi Y.J.: Need perfect Standards for RFID frequency (in Chinese), http://industry.ccidnet.com/art/786/20050426/243871_1.htm1

20. Zhang, B.: Application Standards of RFID Technology in logistics Standards of wave frequency (in Chinese),

http://tech.rfidworld.com.cn/2008_10/200810141048387200.html 